\title{
Self-generation by a Mobile Robot of Topological Maps of Corridors
}

\author{
V. Egido, R. Barber, M.J.L. Boada, M.A. Salichs \\ Universidad Carlos III de Madrid, Division of Systems Engineering and Automation. \\ C/ Butarque 15, 28911 Leganés (Madrid) SPAIN \\ \{vegido, rbarber, mjboada, salichs\}@ ing.uc3m.es
}

\begin{abstract}
In the present paper a system for generation of topological maps is going to be presented. This system is considered as one of the deliberative skills of the mobile robots architecture named $A D . A D$ is a two level architecture: deliberative and automatic. Those skills which require high computational time as consequence of high level reasoning are found in the deliberative level, while the automatic level skills interacts with robot sensors and actuators.

The topological map generated with this deliberative skill is the map belonging to the EDN navigation system, and is named Navigation Chart. In the Navigation Chart, the information obtained from the chart is stored as nodes and as edges. Nodes correspond to the sensorial events and edges correspond to the sensorimotor skills.
\end{abstract}

Keywords-Mobile robots, robot skills, robot navigation, environment modeling.

\section{INTRODUCTION}

Humans have always tried to give inanimate elements human skills. We can find examples even in Greek mythology. It was believed that Hefestos a Greek god (Vulcano for Romans) built tripods with wheels, which moved from one point to another following his orders. The technology evolution, has made possible great improvements in the mobile robotics field, trying to turn out fiction into reality. One of those human skills is learning about a region by moving through it. (Some psychologist have defined that problem like "wayfinding"). In this paper it is pretended for a mobile robot to move autonomously in a corridor and learn about it without human help. The learned part about the environment is stored in a topological map with some special features called Navigation Chart.

The skill in charge of the Navigation Chart generation proposed is located in a navigation system that is placed in a new control architecture proposed in [2] called $\mathrm{AD}$ (Automatic Deliberative Architecture), a two level architecture explained in section 2. We can find precedents of the $\mathrm{AD}$ architecture in the three layer architectures developed by Firby, Gat and Bonasso.

James Firby [7] developed an execution system of plans named RAP which is based on a "reactive packages program". In his architecture, three layers can be observed: a planning layer, the RAP executor and a control system. Peter Bonnaso architecture [6] divides the problem of the robot intelligence in three layers, named 3T architecture, investigating the way to combine the deliberative and reactive control to program robots which will be able to perform tasks in a robust way in different environments. Finally, Erann Gat architecture [8] integrates planning and reactivity, using the planning results as a guide, but not for direct control. His architecture has three components: the controller, the sequencer and the deliberator.

On the other hand, another important issue is the navigation system being used. In the mobile robotics field, many authors consider that the living beings way of moving, in an environment, is by topological structures and by topological relationships. The information needed to navigate is stored in topological maps. These maps are made up, in most of the cases, by nodes and edges. Among these navigation systems the EDN (Event Driven Navigation) system proposed by Barber in [3] can be found.

Some authors [9] related nodes to locations defined by the information obtained from the sensors. Kortenkamp and Weymouth [10] use a combination of sonar sensors with visual information to identify the locations. The sensorial information is processed, obtaining marks such as doors, walls, corners, elements with which the different nodes are identified. Park and Kender [11] use a map constructor and a navigator based on topological relationships between marks. Beccari, Caselli, Zanichelli and Diemmi [4] construct and environment representation 
based on the preexistence of structural marks. The detected marks find place in the topological map, only establishing adjacent relationships with the rest of marks.

In this work, the skill in charge of the topological map generation, in the AD architecture, is presented. This skill is located in the deliberative level due to the time needed to process the information from the low level and the time needed to generate the environment map. The navigation system used is EDN and the generated topological map will then be the Navigation Chart. This chart is the environment topological representation by the EDN navigation system.

\section{AD ARQUITECTURE}

The control architecture used in this work is the AD architecture [2]. The motivation of the AD architecture is the human being reasoning capacity and the actuation capacity.

According to the theories of modern psychology, there are two mechanisms for processing information: reflexive processes and the automatic ones. Therefore, two mental levels of activity can be differentiated in human beings: the deliberative level and the automatic one. These two levels are related to how the reasoning capacity and the actuation capacity are distributed.

According to the criteria exposed, only two levels can be established, as is shown in figure 1. The deliberative level is associated with the reflexive processes. The automatic level is the one associated to automatic processes. The deliberative level will be composed by those processes which require a long time of calculation as a consequence of reasoning. The path planner, the environment modelling and the task supervisor are skills which are found in this level. The automatic level is formed by the skills which interact with the sensors and the actuators and which require minimum time to process the information they work with. Among them are modules which provide the sensorial information and the action modules upon the different mechanical elements of the robot can be found.

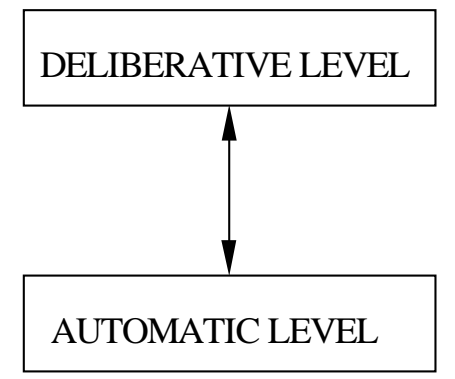

Figure 1. AD architecture levels
Both levels present the same characteristic: they are formed by skills. Skills consist of the different capacities of reasoning or carrying out an action. Those skills are activated by execution orders produced by others skills or by a sequencer. These skills return data and events to the skills or sequencers which have activated them. Those skills are the base of the AD architecture.

\section{A. Deliberative level}

In this level we find modules that require reasoning or decision capacity. Those modules do not produce immediate responses. They need to process the information they work with. Those modules will form the deliberative skills, and they will be activated by a sequencer, that will be in charge of managing the correct performance of these skills. Figure 2 shows this level. This level is formed by a series of skills named deliberative skills, a long-term memory where information is obtained as well as a sequencer that activates and deactivates the deliberative skills.

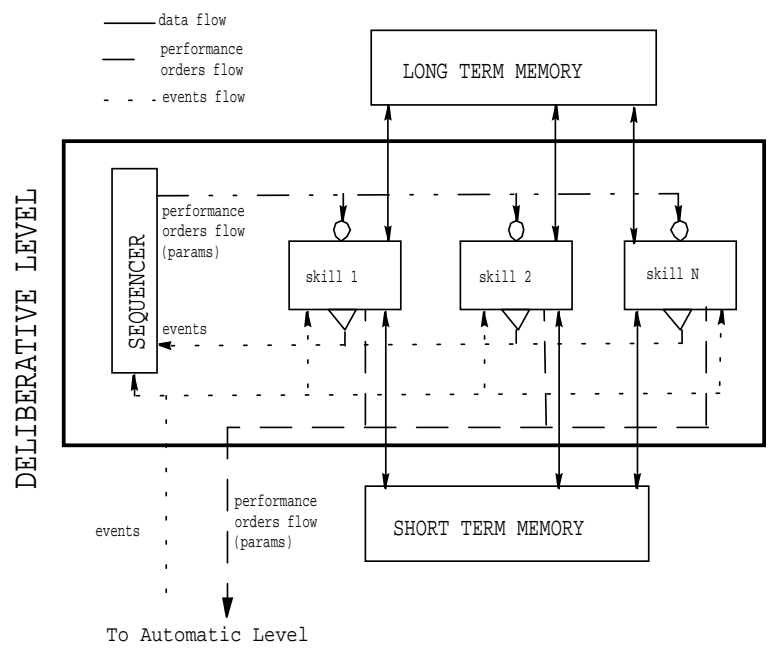

Figure 2. Deliberative level

\section{B. Automatic level}

In this level, there exists low level control modules, which act directly upon the actuators, as well as the modules that collect data from the different sensors of the system. Figure 3 shows the different elements that form the automatic level. The automatic skills are the sensorial and motor capacities of the system. Automatic skills' respond is faster than the deliberative skills' respond. Reflex actions are involuntary and priority responses to a stimulus. The intensity and duration of the response is governed by the intensity and duration of the stimulus. 


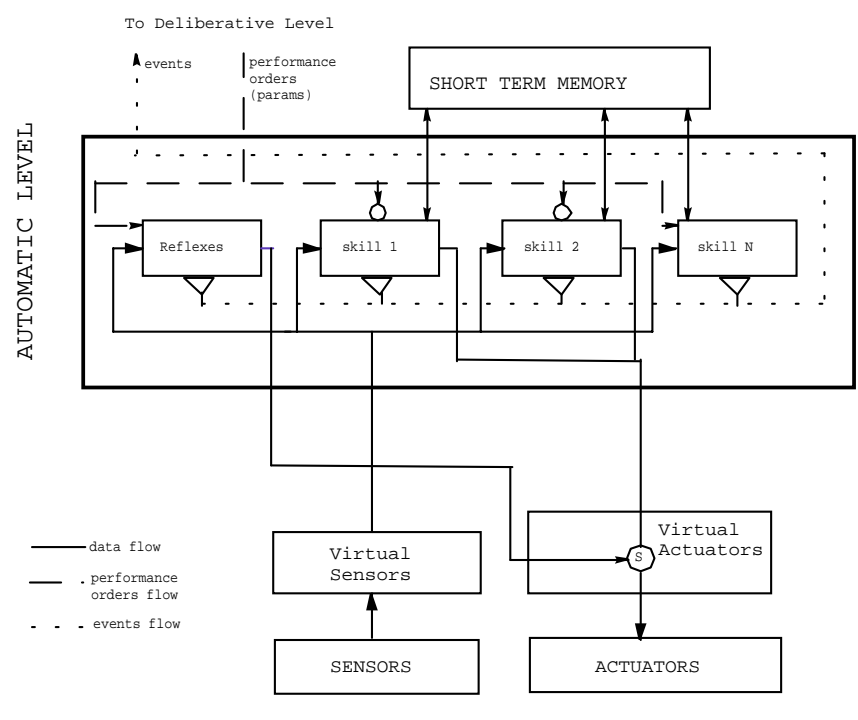

Figure 3. Automatic level

\section{Communication between deliberative and automatic level}

A bidirectional communication between deliberative level and automatic level exists. The deliberative level communicates to the automatic level the execution orders of the automatic skills. It also transfers data at the lower level by the short-term memory. For example the parameters needed to execute some of the automatic skills.

\section{EDN NAVIAGATION SYSTEM}

On this paper the EDN system [3] is used. The EDN system consists of sensorimotor actions and sensorial events which guide the robot to its final destination. The actions and sensorial events succession is also used to explore the environment. By using a succession of similar actions to those a human being will perform, we intend to explore an unknown environment acquiring a spatial knowledge of itself. For example, if a person wants to begin a new exploration from a room, the actions he will perform will be the following:

- Search for a door and exit the room.

- Travel a corridor locating the existing doors on it.

- Move to a door and cross it.

This map, which is not formed by a succession of places of the environment but by a succession of elementary skills is named [3] Navigation Chart. The Navigation Chart is a succession of nodes and edges. Edges represent sensorimotor skills which indicate the robot what to do (move towards a door) and nodes will be events (be in front of a door) which will lead to a new sensorimotor action. Figure 4 shows an example with a topological graph as the one considered in the Navigation Chart.

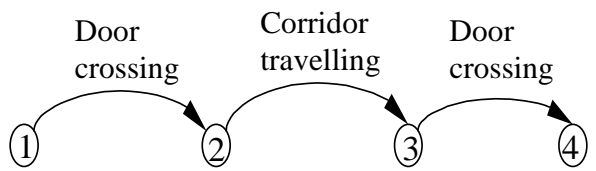

Door detected Corridor Door detected Destination on the left detected on the right detected

Figure 4. Topological graph considered on the Navigation Chart.

Nodes are defined by sensorial perceptions, meaning that nodes are what the robot is sensing in that moment. For example, a node can be: being place underneath a door, door detected on the right, or detection a corridor crossroad.

Edges or transitions are defined by motor actions. Transitions between nodes are performed by robot actions or robot movements. Each action is performed by a predominant skill which can either be simple or complex. A transition can then be actions such as turn right, follow a corridor, move until door detect or cross a door. Edges are related to motor skills, being this the one that defines the edge.

The following work will describe how a robot can explore the environment which surrounds it and at the same time how Navigation Chart information will be stored using the EDN system.

\section{AUTONOMOUS GENERATION OF A CORRIDOR NAVIGATION CHART.}

In this section a deliberative skill which is able to generate a Navigation Chart of a corridor autonomously will be described.

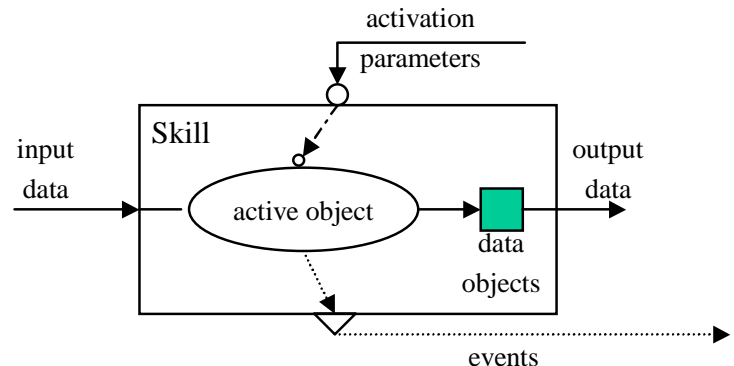

Figure 5. Skill's structure

The structure of the skill is based on the definition given by Boada in [5], where skills are defined as the capacity of processing sensorial information and/or 


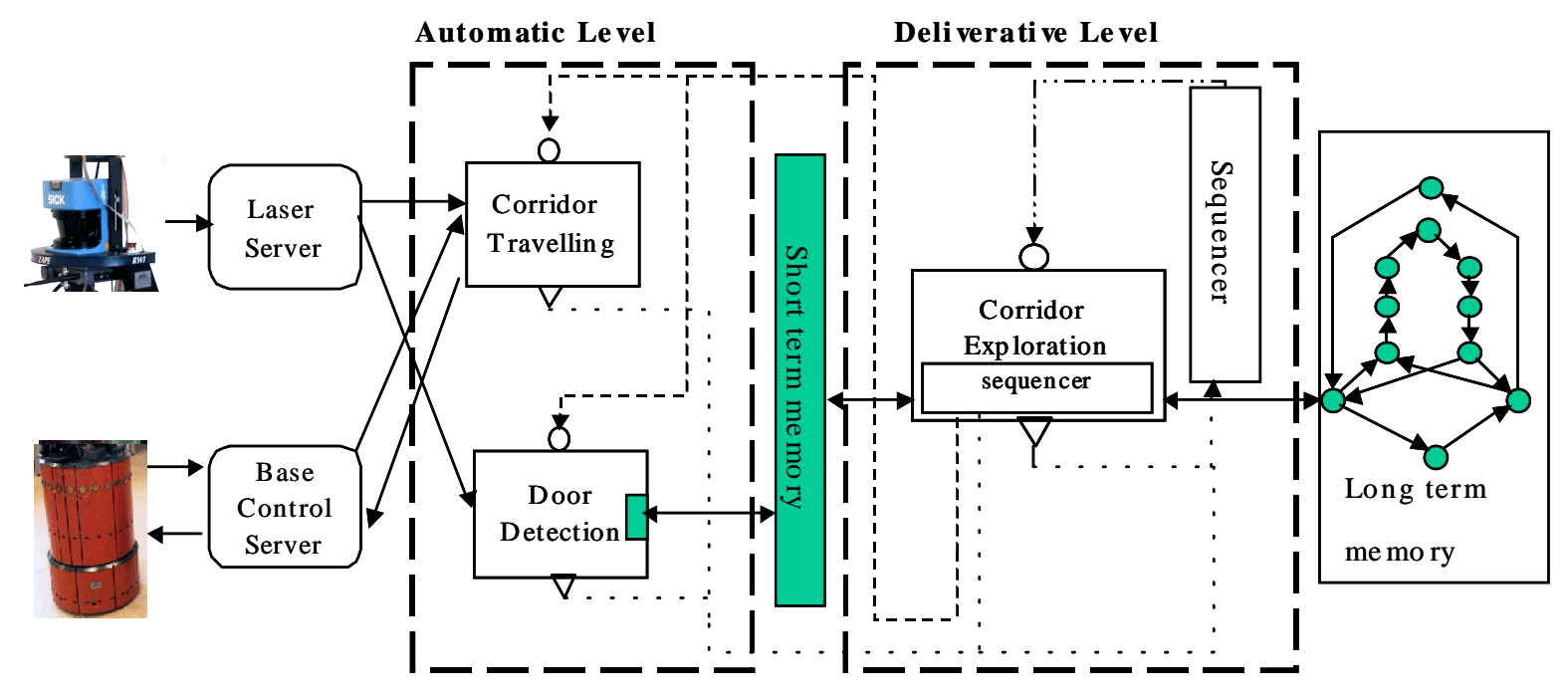

Figure 6. Structure of the AD architecture with the "Corridor Exploration" skill.

executing actions over the robot actuators. As it is described, skills are server/client modules and its structure is shown in figure 5.

Based on the $\mathrm{AD}$ architecture, the skill in charge of the topological map generation of a corridor "Corridor exploration" is located in the deliberative level, as it is shown in figure 6.

The "Corridor exploration" skill is activated and the robot navigates down the corridor, avoiding any obstacle it finds in its way and registering the doors detected at both sides. With this information, the "Corridor exploration" skill will generate the Navigation Chart of the corridor and will store it in the long term memory. This will allow future navigation, down the corridor, using the Navigation Chart previously stored.

\section{A. Corridor Travelling}

This skill is a complex sensorimotor skill. It is a sensorimotor skill because it perceives the information from sensors, perceptive skills or other sensorimotor skills and based on this information it performs an action over the actuators [5].

It is a complex skill because is formed by sequencing four different automatic skills, "Centered travelling", "Contour following", "Avoid obstacles" and "Free space". We can obtain complex skills by three different methods proposed by Barber in [1]: sequencing, output addition and data flow.

Sequencing the four different skills, the robot will navigate down the corridor, avoiding any obstacle it finds in its way.

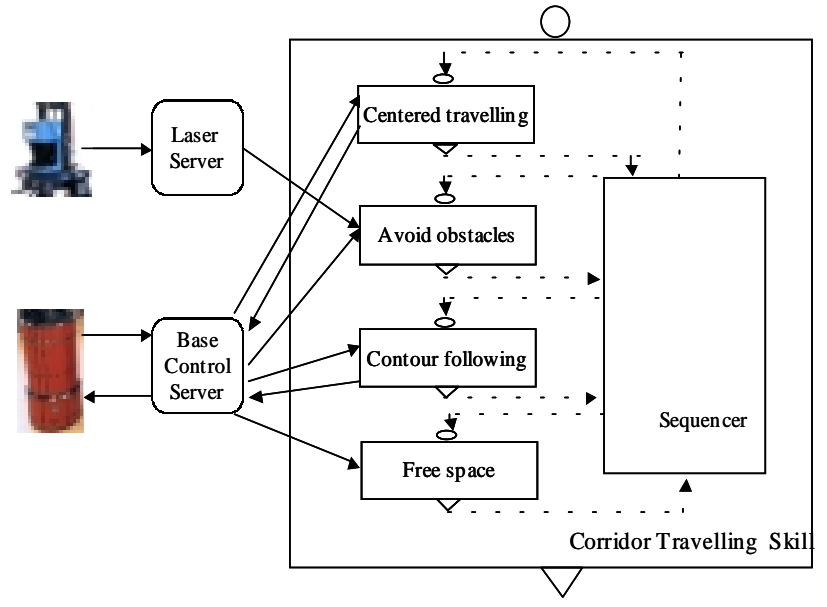

Figure 7. "Corridor travelling" skill.

First, the "Centered travelling" skill and the "Avoid obstacles" skill are activated, the "Centered travelling" skill allows the robot to navigate along the corridor parallel to the walls. Figure 8 shows some variables of the skill.

The "Avoid obstacles" skill identifies any possible obstacle, for the robot navigation in the environment. If the end of the corridor is reached the skill is completed, but if an obstacle appears, then the "Centered travelling" skill is deactivated and the "Contour following" skill is activated. When the robot is able to navigate down the corridor again, the "Free space" skill generates an event and then the "Centered travelling" skill and the "Avoid obstacles" skill are reactivated. 


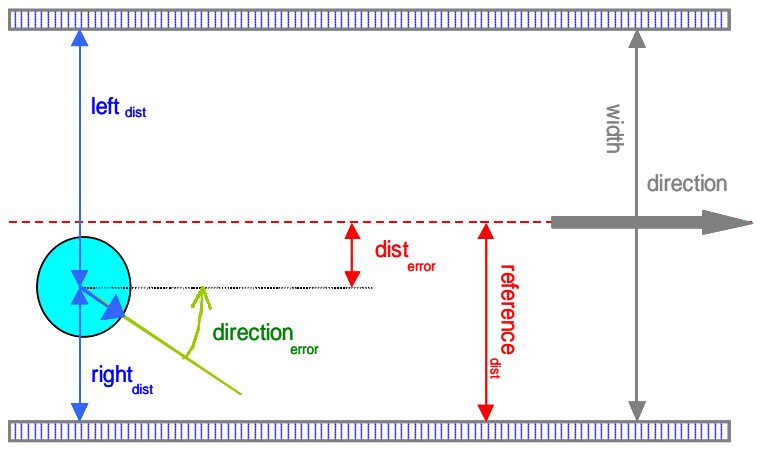

Figure 8. "Centered traveling" skill variables

\section{B. Door Detection}

The "Door detection" skill uses the laser information to detect doors across the corridor at both sides. Using the Hough transform it considers the different segments formed by the information obtained with the laser. With the length of the segments (which determine the width of the doors) and its orientation, the different doors are detected. The information given by this skill is the distance, the orientation and the direction of the door. Figure 9 shows a laser frame taken in a corridor and the different segments calculated.

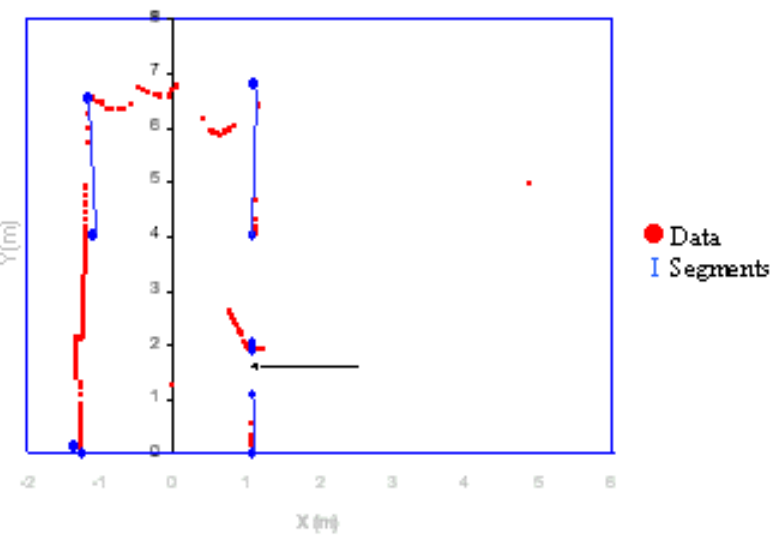

Figure 9. A laser frame and the different segments calculated.

\section{EXPERIMENTAL RESULTS}

The experiments described in this section have been tested in a B21 robot by RWI, as it is shown in figure 10, equipped with a laser scanner PLS-222 from SICK. The maximum number of measurements values transferred by this laser is 361 with an angular resolution of $0.5^{\circ}$.

The skills and sequencers, previously mentioned, have been implemented in $\mathrm{C}++$ language, using the system specified by CORBA which provides interoperability between objects in a heterogeneous, distributed environment.

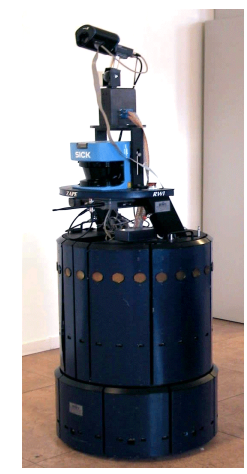

Figure 10. B21 robot with a laser telemeter

The experiment took place in a corridor with five opened doors. Its mission was to start from one end of the corridor, navigate down the corridor, and obtain the corridor navigation chart autonomously. Figure 11 shows a representation of the two paths followed by the robot.
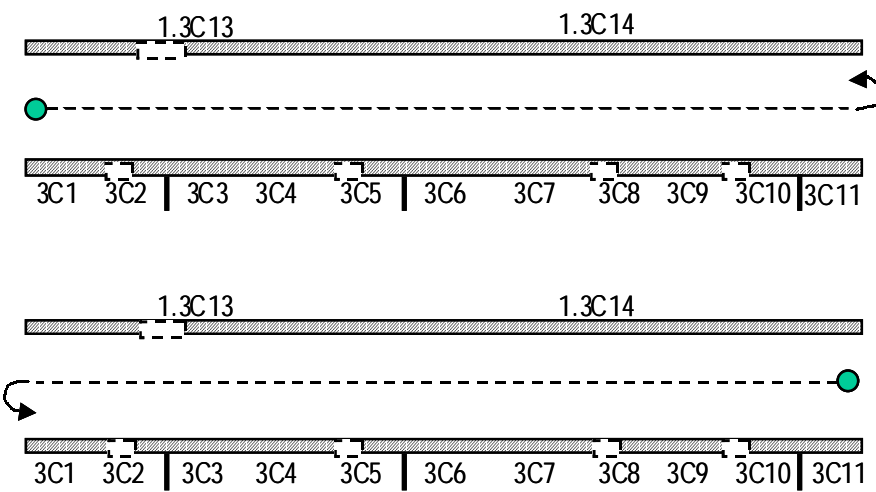

Figure 11. Representation of the two paths followed by the robot.

The end in which the robot begins the corridor travelling does not interfere with the corridor navigation chart. The instructions generated by the navigation chart will not be different if the robot goes from the left side of the corridor to the right side of it or vice versa. For this reason the map generation in both ways is considered, when the experiments for the analysis of the system robustness were carried out.

\begin{tabular}{|c|c|c|c|c|}
\hline & $1^{\mathbf{o}}$ & $2^{\mathbf{o}}$ & $3^{\mathbf{0}}$ & $4^{\mathbf{0}}$ \\
\hline Time(s) & 65 & 70 & 66 & 67 \\
\hline $\begin{array}{c}\text { Sequence } \\
\text { of rooms }\end{array}$ & $\mathrm{C} 2, \mathrm{C} 13$, & $\mathrm{C} 10, \mathrm{C} 8, \mathrm{C} 5$ & $\mathrm{C} 2, \mathrm{C} 13, \mathrm{C} 10$ & $\mathrm{C} 10, \mathrm{C} 8, \mathrm{C} 5$ \\
$\mathrm{C} 13, \mathrm{C} 2$ & $\mathrm{C} 8, \mathrm{C} 10$ & $\mathrm{C} 13, \mathrm{C} 2$ \\
\hline
\end{tabular}

Table 1. Time and distance results searching for an event 
The Navigation Chart generated is shown in figure 11 TR. means turn right and TL means turn left.

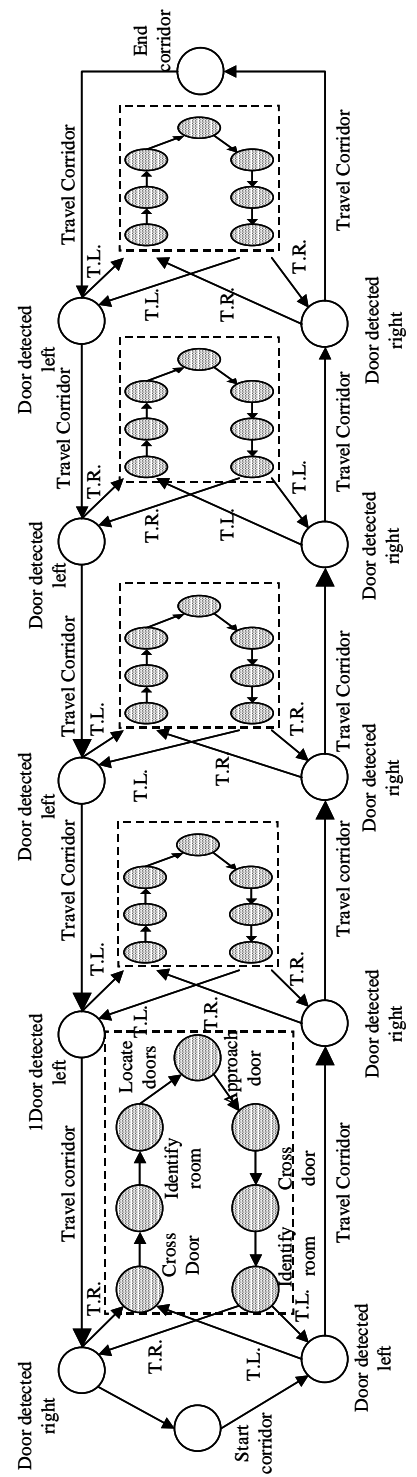

Figure 12. The five rooms corridor navigation chart

\section{ACKNOWLEDGES}

The authors gratefully acknowledge the funds provided by the Spanish Government through the CICYT project TAP99-0214.

\section{REFERENCES}

[1] Barber, R. (2000) "Desarrollo de una Arquitectura para Robots Móviles Autónomos. Aplicación a un Sistema de Navegación Topológica" $\mathrm{PhD}$ Thesis, Universidad Carlos III de Madrid.

[2] Barber, R., Salichs, M.A. (2001a) "A new human based architecture for intelligent autonomous robots". The Fourth IFAC Symposium on Intelligent Autonomous Vehicles, pP85-90.

[3] Barber, R., Salichs, M. A. (2001b) "Mobile Robot Navigation Based on Event Maps”. Field and Service Robotics, p61-66.

[4] Beccari, G.; Caselli, S.; Zanichelli, F.; Diemmi, D. (1997) "Inducing topological maps from task-oriented perception and exploratory behaviors." Proceedings Second EUROMICRO Workshop on Advanced Mobile Robots. p. 134-140. IEEE Comput. Soc. USA.

[5] Boada, M. J. L., Salichs, M. A. (2001) “ Visual Approach Skill for a Mobile Robot using Learning and Fusion of Simple Skills". 9 ${ }^{\text {th }}$ European Workshop on Learning Robots, EWLR-9. Prague, p110-118.

[6] Bonasso, R.P., Firby, J., Gat, E.,Kortenkamp, D., Miller,D.P., Slack, M. G. (1997) "Experiences with an Architecture for Inteligents Reactive Agents". Journal of Experimental Theory of Artificial Intelligence. Vol 9, p237-256

[7] Firby, Jammes R. (1994) "Task Networks for Controlling Continuous Processes". In sensorial International Conference on AI Planning Systems. Ed Kristian Hammond, p 49-54.Menlo Park, California. AAAI Press.

[8] Gat, E. (1992) "Integrating Planning and Reacting in a Heterogeneous Asynchronous Architecture for controlling Real-World Mobile Robots". In Tenth National Conference on Artificial Intelligence. AAAI. San Jose, CA.

[9] Franz, M.O.; Scholkopf, B.; Mallot, H.A.; Bulhoff, H.H. (1998) "Learning view graphs for robot navigation." Autonomous Robots. Vol. 5, Iss. 1, p. 111-25. Kluwer Academic Publishers. Netherlands.

[10] Kortenkamp, D.; Weymouth, T. (1994) "Topological mapping for mobile robots using a combination of sonar and vision sensing." Proceeding of the 12th National Conference on Artificial Intelligence. Vol. 2, p. 979-84. MIT Press. USA.

[11] Park, Il-Pyung; Kender, J.R. (1995) "Topological direction-giving and visual navigation in large enviroments. " Artificial Intelligence. Vol. 78, Iss. 1-2, p. 355-395. Elsevier. Netherlands. 